\title{
Professor Walter neuer DGZMK-Präsident
}

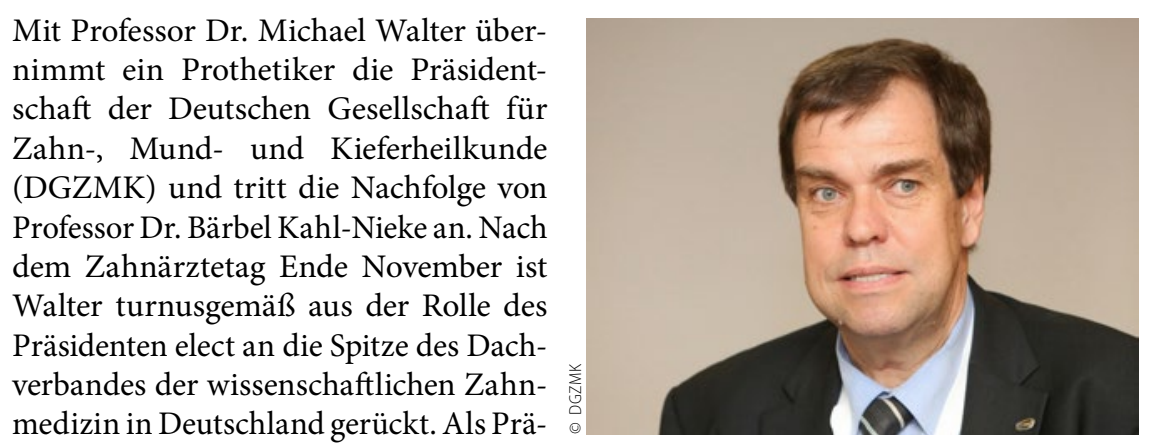

sident der über 22.000 Mitglieder starken Dr. Michael Walter zin und Biomaterialien gesammelt. Seine aktuellen Arbeits- und Forschungsschwerpunkte liegen in den Bereichen Versorgungsforschung und klinische Therapiestudien.

Walter sieht bei der Positionierung der Zahn-, Mund- und Kieferheilkunde in der Medizin „die weitere Festigung ihrer Stellung als medizinische Disziplin auf Augenhöhe" als große Herausforderung. „Ganz wichtig ist mir auch, der immer weiter voranschreitenden Kommerzialisierung der Zahnmedizin entgegenzutreten, bei allem Verständnis für die Ursachen dieses Trends“, erklärt Walter. Die Zahnmedizin der Zukunft solle patientenorientiert, individuell und wissenschaftlich begründet sein. „Dafür möchte ich mich stark machen in meiner Tätigkeit als Hochschullehrer in Dresden, aber auch ganz besonders als Präsident der DGZMK.“

\section{Erratum im GOZ-Navigator}

\section{Fehler bei den Leistungen 4050, 4055 und 4150}

Der GOZ-Navigator, den der Freie Verband Deutscher Zahnärzte exklusiv für Mitglieder erstellt hat, ist in vielen Praxen ein wichtiges Hilfsmittel zur Kalkulation von GOZ-Leistungen geworden. Er bietet einen schnell zu erfassenden Vergleich der Honorierung von annähernd vergleichbaren BEMA- und GOZ-Leistungen.

Leider ist bei den Leistungen 4050, 4055 und 4150 ein Fehler unterlaufen: Anstatt pro Zahn, wie in der Gebührenordnung ausgewiesen, wurde auch in der GOZ das Honorar für das gesamte Gebiss zum Vergleich herangezogen. Dabei ergab sich ein extrem hoher Steigerungsfaktor. Das ist natürlich falsch. Ein aufmerksamer Anwender hat darauf hingewiesen. Unten ist zu

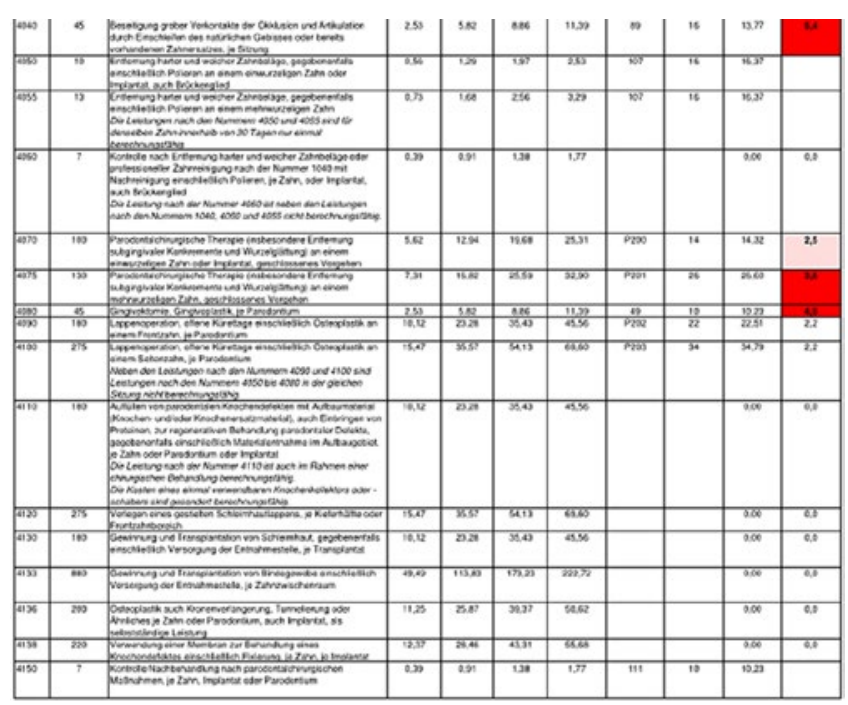

sehen, wie die entsprechenden Seiten im GOZ-Navigator verändert werden müssen, um den Fehler zu korrigieren. Am einfachsten ist es, weiße Papieretiketten auf die rot markierten Faktorfelder in der letzten Spalte der Tabelle zu kleben, so dass sie in Weiß erscheinen. Es steht dann im Feld Steigerungsfaktor keine Zahl mehr, da die Leistungen nicht vergleichbar sind. Der Freie Verband entschuldigt sich für diesen Fehler und dankt für das Verständnis.

Achtung: Bei jeder Leistung sind die durch den Wortlaut der Gebührenordnung im Originaltext vorgegebenen Einschränkungen zu beachten. Öfter mal reinzuschauen, kann nicht schaden und öffnet manchmal die Augen. Die aktuelle GOZ ist auch auf den Seiten des Bundesjustizministeriums (www.gesetze-iminternet.de) oder der Bundeszahnärztekammer (www.bzaek.de) zu finden. Bei der Erstellung von GOZ-Rechnungen sollte der Zahnarzt immer darauf zurückgreifen können.

Von Erstattungsstellen gerne beanstandet werden Beschränkungen je Zahn, Region oder Operationsfeld oder auch die Vorgabe von Mindestzeiten. Neue elektronische Erfassungen erlauben das schelle gezielte Scannen nach typischen Fehlern und Stichworten. Daher kommen vermehrt Anfragen der Erstattungsstellen und der Patienten, die sich direkt auf den Wortlaut der Verordnung beziehen. So wird gezielt nach dem Zeitaufwand, hier im Besonderen die GOÄ Ä3 (mindestens zehn Minuten), die GOZ 1000 (mindestens 25 Minuten) und GOZ 1010 (mindestens 15 Minuten), oder dem Ausführenden der Behandlung gefragt, oder ob der Zahnarzt die Leistung angeordnet und kontrolliert und den Delegationsvorgaben Rechnung getragen hat. Wer sich an den genauen Wortlaut der GOZ hält, ist auf der sicheren Seite.

Dr. Christian Öttl, Mitglied im FVDZ-Bundesvorstand 